\title{
Vaginal epidermal dendritic cells: defense against HIV-1 or a safe haven?
}

\author{
Stephan Caucheteux ${ }^{1}$ and Vincent Piguet ${ }^{1,2}$ \\ 'Division of Dermatology, Department of Medicine, University of Toronto, Toronto, Ontario, Canada. ²Division of Dermatology, Women's College Hospital, Toronto, Ontario, Canada.
}

\begin{abstract}
Langerhans cells (LCs) are likely among the first targets of HIV-1 infection due to their localization in mucosal tissues. In their recent work, Pena-Cruz and colleagues were able to study HIV-1 infection in vaginal epithelial DCs (VEDCs), termed CD1a+ VEDCs. They show that VEDCs are distinct from other blood- and tissue-derived DCs or LCs because they express the protein langerin but not the lectin receptor DC-SICN, and they do not have Birbeck granules. The results from this study indicate that HIV-1 using CXCR4 replicates poorly in VEDCs but that a higher replication for HIV-1 using CCR5 strains is supported by VDECs. Furthermore, Pena-Cruz and colleagues demonstrate that VDECs can represent a viral reservoir in HIV-1-infected virologically suppressed women. As such, VDECs may represent another sanctuary of viral persistence and can be an additional obstacle to viral eradication.
\end{abstract}

(10). HIV-1 viral proteins and nucleic acids can be detected in DCs and their subsets by pattern-recognition receptors such as TLRs and C-type lectins. For instance, the HIV envelope can activate conventional DCs through mTOR signaling, impairing autophagy, and TLR4 pathways (11). Interestingly, LCs seem relatively resistant to HIV infection, in part due to entry and postentry mechanisms. This includes blocks induced by Langerin (12), as well as restriction factor Trim $5 \alpha(13,14)$ and TGF- $\beta$-mediated postentry restrictions to HIV infection (15).

Low levels of HIV-1 viremia not only have a role in early HIV infection, but those levels can be maintained and detected in the plasma of patients for many years during the later phase of HIV-1 infection. The low division rate of DCs may account for the long-term maintenance of the virus and the establishment of a latent reservoir that can persist despite antiretroviral treatment. The ability of HIV-1 to propagate from DCs to $\mathrm{CD}^{+} \mathrm{T}$ cells via infectious or virological synapses (16 and reviewed in ref. 17) make DCs ideal candidates for intervention during both the initial and late stages of infection.

In high-risk populations, pre-exposure prophylaxis (PrEP) has shown promising results by treating noninfected individuals with antiretroviral drugs to prevent early HIV-1 infection, particularly in men at high risk (18). In contrast, tenofovir-based PrEP for HIV infection among African women was unable to reduce the rates of HIV-1 acquisition in an intention-to-treat analysis, probably due to low adherence to study drugs (19). Understanding early events of HIV-1 infection, including infection in vaginal subsets of DCs, may hold answers to some of the challenges of preventing sexual transmission of HIV-1.

Identification of a unique DC subtype opens up doors toward new therapeutic strategies

In the current issue of the JCI (20), PenaCruz and colleagues have identified and 


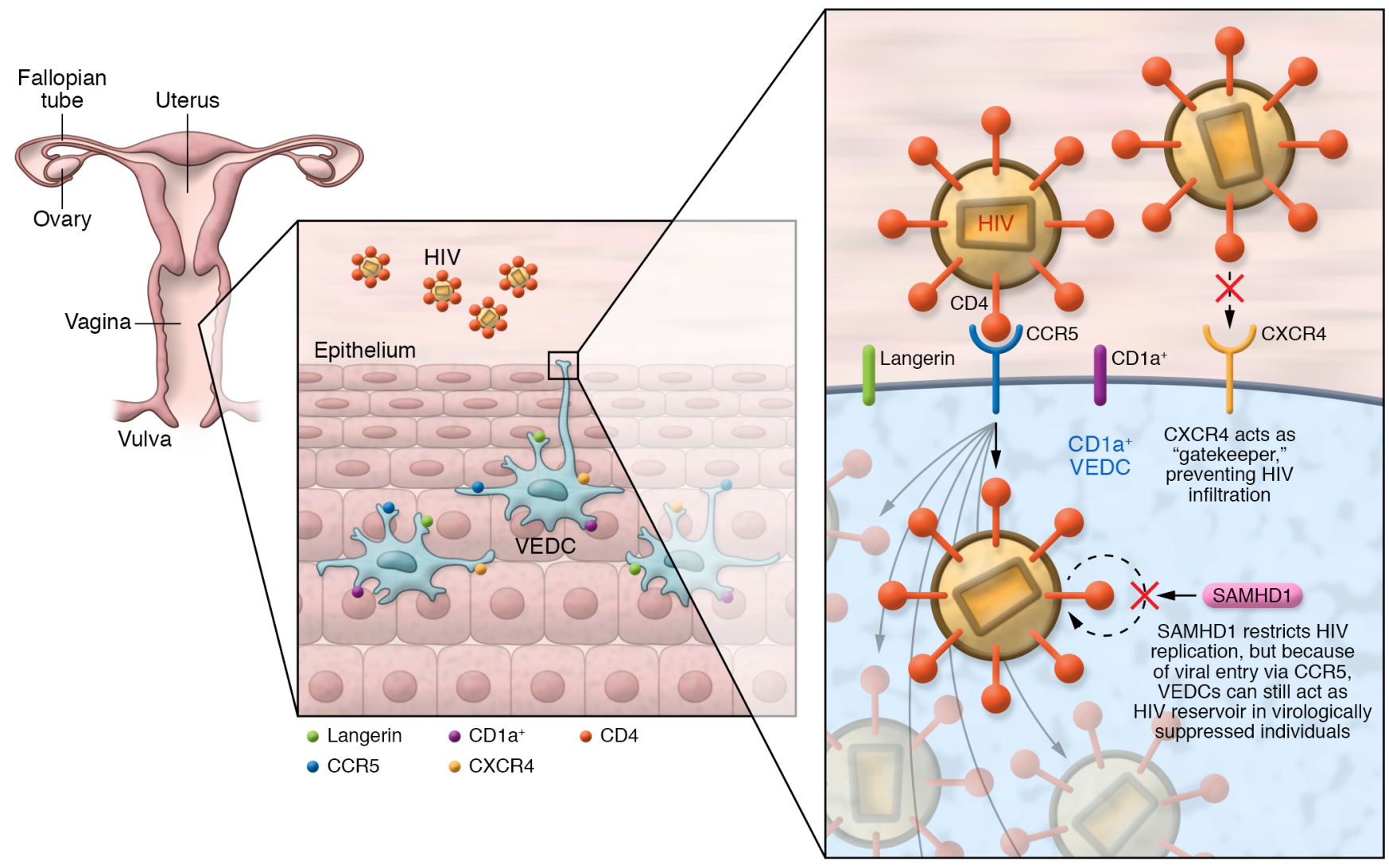

Figure 1. VDECs and HIV-1. CD1a+ VEDCs are purified from the vaginal epithelium. VEDCs preferentially replicate HIV-1 using CCR5. VEDCs express SAMHD1, which acts as a cellular restriction to HIV-1. However, although VEDCs can be gatekeepers by not being infected efficiently by CXCR4-using HIV-1 strains, they can contain virus even in virologically suppressed women and are therefore potential reservoirs for the virus.

described an apparently unique subset of VEDCs that shares characteristics found in skin DCs and in LCs but which remains distinct from the other well-known DC and LC subsets. These cells, labeled $\mathrm{CD} 1^{+} \mathrm{VEDCs}$, display a unique phenotype wherein they express langerin and CD1a, but not DC-SIGN, and do not harbor Birkbeck granules like LCs do. VEDCs express the HIV-1 coreceptors CXCR4 and CCR5 just like mucosal-derived DCs and LCs, yet they are more susceptible to HIV-1 infection by CCR5-using strains as compared with CXCR4-using strains. Furthermore, the authors show that VDECs express host restriction factors to HIV-1 infection, such as dendritic and myeloid cell-specific HIV-1 restriction factor SAMHD1 (21). Importantly, the authors also show that the HIV-1 DNA was detectable in VEDCs isolated from two HIV-1-infected virologically suppressed women, thereby indicating that VEDCs may act as a potential reservoir during latency.

These intriguing observations open up a number of questions for the field.
Are VEDCs a unique cell type? Do they differ from LCs in the male foreskin? Perhaps new techniques such as unbiased single-cell RNA sequencing would shine light on these questions. Another problem with ex vivo human cell systems such as the one used in the study by Pena-Cruz et al. is that extraction and purification of the LCs will potentially alter their phenotype. How much of the process has affected the results obtained? Are these cells truly different from their more-studied counterpart, skin-derived LCs? Additionally, although the authors show that SAMHD1 plays a role as a restriction factor in VEDCs, the contribution of additional potential restrictions such as langerin, Trim $5 \alpha$, and others needs to be explored. Preventing HIV transmission remains a great unmet challenge, particularly in women (19). Are VEDCs another obstacle to HIV-1 infection or do they represent a viral reservoir that has already been accounted for? Only by having clear answers to these questions will we be able to develop novel therapies to prevent HIV infection at its earliest stages.

\section{Acknowledgments}

We thank the Department of Medicine at the University of Toronto for support of this work and Tessa Catchpole for editorial assistance.

Address correspondence to: Vincent Piguet, Dermatology, Department of Medicine, University of Toronto, Women's College Hospital, 76 Grenville Street, Office 6425, Toronto, Ontario M5S 1B2, Canada. Phone: 416.323.6400 ext 5825; Email: vincent.piguet@utoronto.ca.

1. Piguet V, Steinman RM. The interaction of HIV with dendritic cells: outcomes and pathways. Trends Immunol. 2007;28(11):503-510.

2. Haase AT. Early events in sexual transmission of HIV and SIV and opportunities for interventions. Annu Rev Med. 2011;62:127-139.

3. Ahmed Z, Kawamura T, Shimada S, Piguet V. The role of human dendritic cells in HIV-1 infection. IInvest Dermatol. 2015;135(5):1225-1233.

4. Villani AC, et al. Single-cell RNA-seq reveals new types of human blood dendritic cells, monocytes, and progenitors. Science. 2017;356(6335):eaah4573.

5. Nestle FO, Di Meglio P, Qin JZ, Nickoloff BJ. Skin 
immune sentinels in health and disease. Nat Rev Immunol. 2009;9(10):679-691.

6. Haniffa M, Gunawan M, Jardine L. Human skin dendritic cells in health and disease. J Dermatol Sci. 2015;77(2):85-92.

7. Lutz MB, Strobl H, Schuler G, Romani N. GM-CSF monocyte-derived cells and Langerhans cells as part of the dendritic cell family. Front Immunol. 2017;8:1388.

8. Prodger JL, et al. Chemokine levels in the penile coronal sulcus correlate with HIV-1 acquisition and are reduced by male circumcision in Rakai, Uganda. PLoS Pathog. 2016;12(11):e1006025.

9. Geijtenbeek TB, et al. DC-SIGN, a dendritic cell-specific HIV-1-binding protein that enhances trans-infection of T cells. Cell. 2000;100(5):587-597.

10. Zaitseva M, et al. Expression and function of CCR5 and CXCR4 on human Langerhans cells and macrophages: implications for HIV primary infection. Nat Med. 1997;3(12):1369-1375

11. Blanchet FP, et al. Human immunodeficiency virus-1 inhibition of immunoamphisomes in dendritic cells impairs early innate and adaptive immune responses. Immunity. 2010;32(5):654-669.

12. de Witte $\mathrm{L}$, et al. Langerin is a natural barrier to HIV-1 transmission by Langerhans cells. Nat Med. 2007;13(3):367-371.

13. Pertel T, et al. TRIM5 is an innate immune sensor for the retrovirus capsid lattice. Nature. 2011;472(7343):361-365.

14. Ribeiro CM, et al. Receptor usage dictates HIV-1 restriction by human TRIM $5 \alpha$ in dendritic cell subsets. Nature. 2016;540(7633):448-452.

15. Czubala MA, et al. TGF $\beta$ induces a SAMHD1independent post-entry restriction to HIV-1 infection of human epithelial Langerhans cells. J Invest Dermatol. 2016;136(10):1981-1989.
16. McDonald D, Wu L, Bohks SM, KewalRamani VN, Unutmaz D, Hope TJ. Recruitment of HIV and its receptors to dendritic cell-T cell junctions. Science. 2003;300(5623):1295-1297.

17. Piguet V, Sattentau Q. Dangerous liaisons at the virological synapse. J Clin Invest. 2004;114(5):605-610.

18. Molina JM, et al. On-demand preexposure prophylaxis in men at high risk for HIV-1 infection. N Engl J Med. 2015;373(23):2237-2246.

19. Marrazzo JM, et al. Tenofovir-based preexposure prophylaxis for HIV infection among African women. N Engl J Med. 2015;372(6):509-518.

20. Pena-Cruz V, et al. HIV-1 replicates and persists in vaginal epithelial dendritic cells. JClin Invest. 2018;128(8):3439-3444.

21. Laguette N, et al. SAMHD1 is the dendriticand myeloid-cell-specific HIV-1 restriction factor counteracted by Vpx. Nature. 2011;474(7353):654-657. 